\title{
Acerca de la formación argumentativa en el sistema educacional chileno
}

\author{
About Argumentative Training in the Chilean Educational System \\ Acerca da formação argumentativa no sistema educacional chileno
}

Eduardo German Risco Del Valle ${ }^{1}$

\section{Resumen}

Este trabajo busca, desde una perspectiva académica, pero también desde la experiencia de su autor como profesor de Lengua Castellana en la Enseñanza Media, suministrar un panorama de la enseñanza de la argumentación en el sistema educacional chileno y ofrecer unos subsidios conceptuales que contribuyan a valorar esa misma formación. La comunicación ha sido organizada en tres secciones: una aproximación a la relevancia de la argumentación en la formación ciudadana, la presentación de algunos conceptos básicos sobre argumentación y, finalmente, una descripción del modo en que se presenta este contenido en los textos oficiales del Ministerio de Educación de Chile (Estándares de Formación de Profesores y Programas de estudio de la enseñanza media) enfatizando especialmente los silencios conceptuales de aspectos relevantes de la teoría argumentativa. Cierra este trabajo un conjunto de conclusiones en las que puede evidenciarse el contraste existente entre la relevancia que el Ministerio asigna a la formación argumentativa y los exiguos medios que pone al servicio de los profesores para llevarla a cabo.

\section{Palabras clave}

argumentación; estándares de formación; programas de estudio

\section{Abstract}

This paper aims, from an academic perspective, but also from the author's experience as a Professor of Spanish in Secondary Education, to give an overview of the teaching of argumentation in the Chilean educational system and to provide some conceptual subsidies that contribute to assessing that same education. The communication is organized in three sections: an approach to the relevance of argumentation in civic education, the presentation of some basic concepts about argumentation, and, finally, a description of the way this content is presented in the official texts of the Ministry of Education of Chile (Teacher Training Standards and Middle School Study Programs), especially emphasizing the conceptual silences of relevant aspects of the argumentative theory. This work ends with a set of conclusions that show the contrast between the relevance that the Ministry assigns to argumentative training and the meager means placed at the service of teachers to carry it out.

\section{Keywords}

argumentation; training standards; study programs

\section{Resumo}

Este trabalho visa apresentar um panorama do ensino da argumentação no sistema educacional chileno e oferecer uma ajuda conceitual que possa contribuir à valoração desta forma de educação, não só desde uma perspectiva acadêmica, mas também desde a experiência de seu autor como professor de Língua Castelhana no Ensino Médio. A comunicação foi organizada em três seções: uma aproximação à relevância da argumentação na formação cidadã, a apresentação de alguns conceitos básicos sobre argumentação e, finalmente, uma descrição da forma na que é apresentado este conteúdo nos textos oficiais do Ministério da Educação de Chile (Estándares de Formação de Professores e Programas de estudo do Ensino Médio) fazendo ênfase nos silêncios conceituais de aspectos relevantes da teoria argumentativa. Para finalizar este trabalho, apresenta-se um conjunto de conclusões nas que é possível evidenciar o contraste existente entre a relevância que o Ministério atribui à formação argumentativa e os exíguos médios que oferece aos professores para efetuá-la.

\section{Palavras-chave}

argumentação; estándares de formação; programas de estudo

Artículo recibido el 7 de mayo de 2017 y aprobado el 10 de enero de 2018

1 Universidad de los Lagos, Chile. Correo electrónico: erisco@ulagos.cl 


\section{Introducción: centralidad de la argumentación en la formación del futuro ciudadano}

Si nos es lícito considerar que la ciudadanía es una realidad que emerge de la relación intersubjetiva que caracteriza a la vida cotidiana de las comunidades, entonces resultará del todo lógico que la práctica de la argumentación haya ocupado siempre un lugar preponderante en la formación de un individuo que, se espera, se integre activamente a la comunidad de la que forma parte.

En los albores de la cultura occidental, en su vertiente griega, ya encontramos la centralidad de la formación argumentativa no solo en cuanto es un contenido más del currículum (constituido por lo que más tarde se llamará el trivium), sino incluso en la forma de un verdadero método educativo diseñado en el seno de la sofística, plasmado en la forma en que los sofistas entendían lo que hoy llamamos retórica clásica y representado de manera más prístina en el pensamiento de Isócrates ${ }^{2}$, a quien Jaeger sitúa en uno de los extremos de las influencias que dieron forma al pensamiento pedagógico occidental. La razón para asignar semejante importancia a la retórica clásica reposa menos en el entusiasmo con el que los sofistas defendieron sus ideas ante las arremetidas platónicas que en la racionalidad interna del pensamiento de la época; el que, teniendo en cuenta el hecho de que no es posible hablar de las cosas sin conocerlas, entiende el ejercicio retórico-argumentativo en el marco del principio que ve a la armonía (relación de las partes con el todo) como la forma natural de existir en el cosmos con lo que la conjunción de ambos presupuestos da en la comprensión de la retórica como un ámbito de integración de todos los conocimientos y de ahí su postulación como método pedagógico global. Para Jaeger (1946): "La retórica constituye la forma predominante de la educación en los últimos tiempos de la antigüedad [...]. En unión de la

2 Atenas 436-338 a. de C. Es considerado uno de los "oradores antiguos" ejemplares por Dionisio de Halicarnaso, quien lo incardina en la categoría de los oradores antiguos, ubicados en la época preclásica de la cultura griega. gramática y la dialéctica, la retórica se convirtió en el fundamento de la formación formal del mundo occidental" (p. 331).

Aunque la intrínseca asociación entre argumentación y retórica (que caracterizó a la retórica clásica) conoció un proceso de distanciamiento en el que "lo que originalmente era (o parecía) un mismo cuerpo de conocimientos se separó en dos" (Risco, 2015 , p. 84 ), lo cierto es que, aun por separado, ambos componentes del método pedagógico de los sofistas continuaron siendo relevantes para la formación de los nuevos ciudadanos a lo largo de un continuum histórico que, con mayores o menores énfasis, llega hasta nuestros días y que incluso conoce un repunte desde las obras refundacionales de Perelman y Olbrecht-Tyteca (2000), por un lado, y de Toulmin (1958), por el otro y que manifiesta el hecho que Covarrubias (2003) apunta del siguiente modo: "En los últimos años ha surgido un creciente interés por los estudios sobre la persuasión, no solamente en filosofía y lingüística sino que también en otras disciplinas como el derecho" (p. 13); y es que, como indicaba Plantin (2002) algún tiempo antes:

La argumentación funciona en la vida corriente. En la esfera social, está en contacto con la economía (permite hacer publicidad, ayuda a vender), con lo judicial (procesos), con la política y con el poder. Se argumenta en todas las situaciones en las que existe una alternativa, una posible contestación, en las que hay que justificar(se), en las que hay que comprometerse en la acción y se tiene que tomar la decisión justa. (p. 25).

Una concepción que subraya la omnipresencia (o al menos multipresencia) social de la conducta argumentativa que, de suyo, instala su derecho de ciudadanía en el ámbito de la formación de las nuevas generaciones y desliza también una casi velada mención a su estatus de mecanismo de control y desarrollo de la elaboración cognitiva que el sujeto individual (y social) hace de la experiencia que lo circunda.

Desde una perspectiva nacional e institucional, la centralidad de la formación argumentativa del futuro ciudadano emerge fácilmente del énfasis que los documentos normativos del Ministerio de 
Educación de Chile (Mineduc) ponen en la formación para la participación. A manera de ejemplo, la Ley 20.370 (Ley General de Educación) señala en su artículo $2^{3}$ :

La educación es el proceso de aprendizaje permanente que abarca las distintas etapas de la vida de las personas y que tiene como finalidad alcanzar su desarrollo espiritual, ético, moral, afectivo, intelectual, artístico y físico, mediante la transmisión y el cultivo de valores, conocimientos y destrezas. Se enmarca en el respeto y valoración de los derechos humanos y de las libertades fundamentales, de la diversidad multicultural y de la paz, y de nuestra identidad nacional, capacitando a las personas para conducir su vida en forma plena, para convivir y participar en forma responsable, tolerante, solidaria, democrática y activa en la comunidad, y para trabajar y contribuir al desarrollo del país. (Mineduc, 2009a).

Este texto, como otros que siguen en los artículos 3,9 y 20, por ejemplo, enfatiza el hecho de que la educación involucra todos los aspectos de la vida y que debe preparar a los estudiantes para su incorporación activa, participativa y responsable a la dinámica de la sociedad, participación que, a falta de otras precisiones, entendemos como particularmente expresada en la discusión entre iguales, en el debate argumentado en torno a políticas, propuestas de estas e ideas relacionadas. Este hecho, que la Presentación de las orientaciones para la elaboración del plan de formación ciudadana ofrece en la página del Mineduc, precisa de mejor manera con la indicación de que contar con apoyos técnicos para la formación ciudadana es una necesidad recurrentemente presentada por los establecimientos educacionales y que la centralidad de tal formación es una preocupación fundamental de distintos actores de la sociedad y del propio Ministerio, el que la concibe como: "[...] un pilar de los procesos de enseñanza/aprendizaje de los estudiantes para así avanzar hacia una sociedad democrática y cada vez más enriquecida de valores, respetuosa de los

3 Subrayados nuestros. derechos humanos, del medio ambiente, inclusiva y participativa" (Mineduc, 2016a)

Del mismo modo, en el cuerpo del texto oficial de las Orientaciones, la centralidad de la formación argumentativa aparece en la sección "Ejemplos de actividades que promueven la formación ciudadana", que menciona los Torneos de Debate, actividad que se describe en los siguientes términos:

Se realizará un torneo desde los niveles de $7^{\circ}$ a

$\mathrm{IV}^{\circ}$ medio, en que se traten temas que interese a los jóvenes y niños. Una comisión integrada por docentes, padres y estudiantes del centro de alumnos elaborará las bases del concurso y los protocolos para su ejecución. (Mineduc, 2016b, p. 54).

Actividad para la que se trazan los siguientes objetivos:

- Fomentar en los estudiantes el ejercicio de una ciudadanía crítica, responsable, respetuosa, abierta y creativa.

- Fomentar la participación de los estudiantes en temas de interés público.

Por último y para mayor abundamiento, en el texto de los programas de tercer año medio, la presentación de la tercera unidad, titulada "Diversidad y conflicto: argumentación y diálogo" se indica que "se busca que, a partir de sus lecturas (especialmente de obras dramáticas), los estudiantes comprendan el funcionamiento y el valor social de la argumentación y el diálogo" (Mineduc, 2009b, p. 98).

Establecida, pues, la centralidad de la formación argumentativa para la formación del futuro ciudadano, corresponde ahora examinar el cómo tal centralidad se expresa en dos instrumentos técnicos destinados a asegurar que tal formación será posible en el sistema escolar: el primero de ellos, destinado al uso de las instituciones de formación superior que imparten carreras pedagógicas, son los estándares orientadores para carreras de pedagogía en educación media; y, el segundo, destinado al uso de los maestros en su ejercicio profesional de aula: los programas de estudio de la educación media. Sin embargo, una valoración de estos instrumentos requiere contar con un marco conceptual mínimo 
que, poniendo en relieve componentes básicos del fenómeno argumentativo, permita reconocer los acentos y, sobre todo, los silencios que contienen tanto los estándares como los programas.

\section{La argumentación: una conceptualización básica}

La argumentación puede entenderse, en una primera aproximación general, como una práctica social que, manifestada en un uso particular de la Facultad del Lenguaje y de su expresión más directamente observable: el habla es fundamentalmente una forma de conducta discursiva, es decir, constituye una suerte de puesta en escena en la que intervienen elementos lingüísticos y no lingüísticos y cuya naturaleza - precisamente por lo que acabamos de afirmar-, excede el campo del análisis lingüístico de la coherencia visible de los elementos formados. Esta práctica social, en ocasiones, puede plasmarse en textos escritos (conocidos bajo el rótulo de texto argumentativo y cuyas reglas específicas de textualización están todavía por ser reconocidas), pero estos no consiguen de ninguna manera captar toda la riqueza de la ocurrencia oral de la práctica discursiva argumental.

Esta práctica social tiene lugar en distintos momentos y escenarios de la vida de una comunidad dada, estos momentos y escenarios pueden ser muy diversos, como lo indica Plantin (2002) en el texto que hemos citado más arriba, pero cuyo evento detonante suele ser siempre el mismo: la existencia de una o más proposiciones ${ }^{4}$ acerca de algo cuya verdad o verosimilitud no resulta evidente para un auditorio determinado y requiere, por tanto, de un ejercicio disquisitivo adicional al que suele implementarse en la comprensión de otras proposiciones. Este esfuerzo disquisitivo se realiza (o debiera realizarse fundamentalmente) sobre la valoración del carácter

4 Utilizamos aquí ex profeso la expresión proposiciones, la que entendemos en su acepción lógica, es decir, un enunciado con la forma "S es P" y que, para el caso que nos ocupa, puede tener la estructura de una oración gramatical, pero que, habiendo suscitado controversia, es lícito pensar que es reductible a la estructura proposicional y que-creemos-es la razón por la que los miembros de un auditorio dado esperan razones justificatorias. de verdad o verosimilitud de la proposición a partir de las razones que la justifican y que aporta el proponente. Van Eemeren et al. (1996), teniendo en cuenta los elementos precedentemente indicados, formulan la siguiente definición:

La argumentación es una actividad verbal y social cuya meta es reforzar o debilitar la aceptabilidad de un punto de vista controvertido ante un oyente o un lector, emitiendo una constelación de proposiciones destinadas a justificar (o a refutar) ese punto de vista ante un juez racional (p. 5).

Esta formulación, además de enfatizar el carácter social de esta conducta verbal, hace explícita la finalidad perseguida por la argumentación, que no es otra que suscitar una determinada conducta en el auditor, cuestión particularmente importante porque suele confundirse esta finalidad con los procedimientos que es posible implementar para alcanzarla. En efecto, dado que el ejercicio argumentativo está destinado a operar -en última instancia- sobre la cognición del destinatario, es posible reconocer dos procedimientos de operación sobre este ámbito: la persuasión (dirigida a la emocionalidad del auditor), y la convicción (dirigida a la racionalidad del auditor), cada uno de ellos condicionado por el grado de estabilidad de la conducta que se pretende suscitar, pues y como es sabido, el procedimiento persuasivo suscita una conducta menos estable que el convincente. Rivano presenta estos procedimientos como fenómenos vinculados a la argumentación y, en una primera formulación atingente al objeto que revisamos aquí, entiende la persuasión como "un proceso pasivo, irracional e irreflexivo por parte del participante paciente" (Rivano, 1999, p. 12), en cuanto entiende la convicción como "un proceso activo, racional y reflexivo por parte participante paciente" (Rivano, 1999, p. 12). Se trata, por supuesto, de una formulación contemporánea de una distinción que ya se encuentra en la tradición de la retórica clásica singularizada en el uso de dos formas verbales griegas distintas para designar ambos procedimientos y atestiguadas ya en la obra de Dionisio de Halicarnaso (Risco, 2015, p. 90), a saber: $\pi \iota \sigma \tau \iota v$ y 


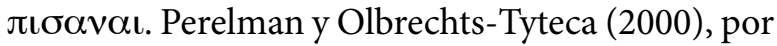
su parte, reinstalan esta distinción:

Para quien se preocupa del resultado, persuadir es más que convencer, no siendo la convicción más que el primer estadio que conduce a la acción. [...] Por el contrario, para quien está preocupado por el carácter racional de la adhesión, convencer es más que persuadir. [...] Nos proponemos llamar persuasiva una argumentación que no pretende ser válida más que para un auditorio particular y llamar convincente a aquella que se entiende destinada a obtener la adhesión de todo ser de razón. (pp. 35-36) .

Estos procedimientos suelen confundirse (y es lo que, desafortunadamente, hacen los autores citados) con las finalidades de la argumentación, pero un análisis un tanto más detallado de sus características nos lleva a concluir que su estatus de procedimientos se justifica en el hecho de que el objetivo de ambos no es otro que suscitar un tipo de conducta $y$ en el hecho de que el logro de sus resultados se da mediante un mismo recurso: los argumentos, que son el recurso con el que se busca alcanzar la finalidad apuntada por Van Eemeren et al. (1996).

En una consideración menos teórica y más pragmática del fenómeno, cuando argumentamos, lo hacemos siempre respecto de algo; ese algo es lo que la tradición ha dado en denominar asunto, tema, objeto o, más recientemente, cuestión del debate y, aunque estas cuestiones podrían parecer a primera vista como infinitas, la verdad es que son reductibles solo a unas cuantas, según lo explica García (2012):

Aunque pudiera parecer que las posibilidades de controversia son infinitas, todas las cuestiones se reducen a tres variedades porque únicamente son tres las dudas o cosas dudosas sobre las que podemos discutir: a. si una cosa existe o no, por ejemplo, si un hecho se ha producido o no. b. en

5 “Pour qui se préoccupe du résultat, persuader est plus que convaincre, la conviction n'étant que le premier stade qui mène à l'action. [...] Par contre, pour qui est préoccupé du caractère rationnel de l'adhésion, convaincre est plus que persuader. [...] Nous nous proposons d'appeler persuasive une argumentation qui ne prétend valoir que pour un auditoire particulier et d'appeler convaincante celle qui est censée obtenir l'adhésion de tout être de raison" (traducción nuestra). qué consiste, esto es, qué nombre le corresponde.

c. si nos parece bien o mal. (p. 14).

Una primera formulación que, no obstante, el mismo autor completa más adelante con un elenco que reordena esta primera distinción en dos categorías: cuestiones de hechos y de acción; entre las primeras reconocemos las tres cuestiones antes señaladas: cuestión conjetural (si los hechos han ocurrido o podrían llegar a ocurrir), cuestión nominal (qué nombre debemos dar a estos hechos, por ejemplo: robo con violencia, robo con intimidación, etc.) y cuestión valorativa (si los hechos acerca de los que debatimos son axiológicamente buenos o malos); entre las segundas, a su vez, reconocemos otros dos tipos de cuestiones: la cuestión deliberativa (qué conviene hacer en el caso) y la cuestión de enjuiciamiento (quién o quienes están involucrados en los hechos debatidos).

El establecimiento de la cuestión acerca de la que se debate, su instalación en el discurso argumentativo y su sostenimiento (argumentativo), por tanto, resulta un aspecto capital en el desarrollo de cualquier debate y permite, entre otras cosas, establecer cuando un argumentador dado se mantiene coherentemente en el desarrollo de una cuestión dada o la abandona instalando una nueva, siendo esperable que, para mantener la coherencia necesaria, no se instale una nueva cuestión sin que se haya resuelto aquella acerca de la cual se debate en primer lugar.

Los medios que permiten el sostenimiento de una cuestión (una vez instalada mediante una proposición conflictiva) son los argumentos; estos pueden definirse como una estructura compuesta por una proposición y las razones que la apoyan ${ }^{6}$, un tipo de razonamiento que, más allá de su forma lingüística, es reductible a una forma lógica silogística o cuasisilogística que facilita su análisis. Para este medio, Van Eemeren, Grotendorst y Snoek (2006)

6 Aunque a este respecto hay que reconocer que no hay consenso, es posible encontrar autores que coinciden con nuestra definición y otros que utilizan el término argumento para indicar exclusivamente a las razones que apoyan una determinada proposición. Nuestra definición reposa en el convencimiento de que el análisis -por reducción silogística del argumento-no permite excluir del mismo la proposición controvertida, a la que Toulmin llama tesis. 
ofrecen la siguiente definición: "Un argumento en su forma más explícita está constituido en dos y solo dos premisas. Usualmente, una de estas es implícitas, de manera que el argumento [...] parece conformarse de una sola premisa" (Van Eemeren, Grootendorst y Snoeck, 2006, pp. 69-70); se trata de una definición confusa porque, en la proposición de un método de análisis de la argumentación que los mismos autores ofrecen en su libro, se acude a una reducción a estructura cuasisilogística que es imposible o, a lo menos, muy difícil a partir de una sola premisa. García (2012), por su parte, proporciona la siguiente definición: "Nosotros llamaremos argumento al conjunto de razones que sostienen una conclusión" (p. 121), lo que también resulta algo confuso y aparentemente incompatible con el método de análisis que el mismo autor utiliza para tales argumentos (reducción a estructura cuasisilogística), razón por la que nos convencemos de que nuestra concepción de argumento (más arriba indicada) constituye la formulación más adecuada y se emparenta con la que insinúa Toulmin en su modelo y que se refiere a una estructura compuesta (cognitivamente al menos) de dos premisas y una conclusión, aceptando que una de estas premisas puede mantenerse implícita.

Desde la tradición de la retórica clásica hasta nuestros días, se ha desplegado un ingente esfuerzo por definir taxonomías estables de formas argumentales, razón por la cual estas conservan muchos de sus nombres en latín; por razones de espacio no las detallaremos aquí, pero remitimos a la propuesta taxonómica que hace García (2012, p. 177).

Sin detrimento de lo anteriormente expuesto, para todo observador de la práctica argumentativa cotidiana resulta notorio que hay una actitud moral básica que todo sujeto involucrado en un evento argumentativo, dado que debe observar si se espera resolver la cuestión controvertida respecto de la que se discute y que es posible formular en el siguiente precepto: "Si no puedo encontrar argumentos en contra de la proposición que defiende mi contraargumentador, debo aceptar que él tiene la razón". Se trata, por supuesto, de una cuestión capital porque la observancia de este precepto asegura que la discusión no pierda su norte y también asegura la pertinencia y licitud de los recursos argumentales desplegados. En efecto, tratándose de interacciones fundamentalmente orales, las discusiones involucran aspectos pragmáticos diversos, entre los que se cuenta el compromiso afectivo que los argumentadores pueden tener con la posición que defienden, pero también y principalmente, su autoimagen positiva y la tendencia natural a defenderla de cualquier posible (o aparente) agresión, esto puede incidir fácilmente en que se desencadene un proceso que Pereda (1994) describe del siguiente modo:

Un punto de vista articula, se dijo, una dirección de la atención. Pero no hay atención sin entrega al objeto atendido y, cuanto más importa lo que se atiende, cuanto más concentrada sea la atención, razonablemente, tanto más tenderá el acto a borrar aquello que no capte el foco de ese atender, en particular, lo que se encuentra en el foco opuesto; de ahí la amenaza potencial en cualquier punto de vista de que la atención se fije sin medida y haga desaparecer los otros puntos de vista, ante todo, haga desaparecer al punto de vista opuesto al que se asume en el momento. (p. 107).

Para esta tendencia, Pereda propone el nombre de vértigo argumental que, cuando se presenta, "desencadena en el argüir un dispositivo de repetición" que transforma la discusión de modo que ya no es una interacción que avance en la resolución de una cuestión, sino más bien un monólogo inconducente en el que se filtran nuevas cuestiones (abandonándose sin resolver la instalada en un principio) y facilita, por lo mismo, la recurrencia a recursos argumentativos ilícitos, rotulados como falacias.

En la perspectiva de conjurar la aparición de los vértigos argumentales, Van Eemeren y Grotendorst (2002, pp. 223-224) proponen diez reglas de la discusión crítica que deben observar quienes participan de un debate, a saber:

1. Las partes no deben impedirse unas a otras el presentar puntos de vista o el ponerlos en duda. 
2. Una parte que presenta un punto de vista está obligada a defenderlo si la otra parte le solicita hacerlo.

3. El ataque de una parte en contra de un punto de vista debe referirse al punto de vista que realmente ha sido presentado por la otra parte.

4. Una parte solo puede defender su punto de vista presentando una argumentación que esté relacionada con ese punto de vista.

5. Una parte no puede presentar algo falsamente como si fuera una premisa dejada implícita por la otra parte, ni puede negar una premisa que él mismo ha dejado implícita.

6. Una parte no puede presentar falsamente una premisa como si fuera un punto de partida aceptado, ni puede negar una premisa que presenta un punto de partida aceptado.

7. Una parte no puede considerar que un punto de vista ha sido defendido concluyentemente, si la defensa no se ha llevado a cabo por medio de un esquema argumentativo apropiado que se haya aplicado correctamente.

8. En su argumentación, las partes solo pueden usar argumentos que sean lógicamente válidos o que sean susceptibles de ser validados explicitando una o más premisas implícitas.

9. Una defensa fallida de un punto de vista debe tener como resultado el que la parte que lo presentó se retracte de él y una defensa concluyente debe tener como resultado el que la otra parte se retracte de sus dudas acerca del punto de vista.

10. Las partes no deben usar formulaciones que resulten insuficientemente claras o confusamente ambiguas, y deben interpretar las formulaciones de la parte contraria tan cuidadosa y tan exactamente como les sea posible.

Por último, el contexto o circunstancia social en la que tiene lugar la discusión organizada según los elementos apuntados es el debate. Se trata, por supuesto, de una noción poco trabajada y, en la práctica escolar al menos, excesivamente influenciada por un tipo de evento televisivo organizado por grandes cadenas con ocasión de unos inminentes comicios electorales y que suele ser, más bien ,una ocasión para que los eventuales candidatos a algún cargo público de elección popular desarrollen un discurso expositivo de sus respectivos programas, sin que ocurra una verdadera discusión, aun cuando suele considerarse como tal incidentales alusiones personales que sus participantes hacen respecto de otro que está presente y que bien pueden identificarse con la conocida falacia ad hominem, cuyo problema fundamental es que, en lugar de centrar los esfuerzos en el argumento del contrario, se centra en descalificar a la persona del contrario. En el ámbito que nos ocupa, entenderemos el debate como un ejercicio racional co-construido y co-gestionado por los intervinientes de los que se trate, centrado en una o más cuestiones establecidas o consensuadas por ellos o por otros, en el que se busca dilucidar estas cuestiones, se procede ordenadamente sosteniendo la cuestión instalada hasta despejarla satisfactoriamente, acción a través de recursos argumentales lícitos y respetando las reglas de la discusión crítica más arriba indicadas. Semejante definición, creemos, integra los componentes básicos de la argumentación que venimos desarrollando y, en su uso pedagógico, facilita la evaluación de la actuación argumentativa de los alumnos con prescindencia de otros aspectos que, aunque involucrados, son marginales a la práctica argumentativa en sí.

Ya, en el tratamiento de los recursos argumentales, como en el de los aspectos éticos involucrados en la práctica argumentativa, hemos deslizado la noción de recursos ilícitos y los hemos asociado a la noción de falacia, corresponde ahora referirnos brevemente a ella.

La falacia, antes que todo, es un recurso argumental que ha rendido incontables servicios a muchos argumentadores a lo largo de la historia; sin embargo, ya desde la más prestigiada tradición clásica se le ha considerado un recurso ilícito y se ha proscrito su utilización en el debate. En términos formales, se trata de un argumento que, teniendo la apariencia de una construcción lógica, manifiesta un error (en la mayoría de los casos, imperceptible) que invalida su consideración como argumento 
desde el punto de vista lógico. Veamos un ejemplo: supóngase el siguiente argumento: "Todo hombre es racional y, como ningún hombre es una mujer, entonces ninguna mujer es racional". Una reducción lógica de este argumento presenta la siguiente estructura:

PREMISA MAYOR: Todo hombre es racional

PREMISA MENOR: Ningún hombre es una mujer

CONCLUSIÓN: Ninguna mujer es racional

Un reconocimiento formal de este silogismo nos lo presenta como reductible a la forma AII, que es una de las más fuertes del silogismo, con lo que resultaría un argumento válido; sin embargo, un análisis semántico más cuidadoso nos permite descubrir que la noción de hombre en la primera premisa (especie humana) no es la misma que la presentada en la premisa menor (varón), por lo que se produce un fenómeno semántico que, entre otras posibilidades, podemos rotular como contradictio in termini, con lo que el razonamiento se transforma en inválido. Sin detrimento de esta fuente de invalidación y atendiendo a que las falacias pueden tener formas diversas y no todas evaluables mediante mecanismos lógicos, lo que en realidad origina su proscripción del elenco de recursos argumentales lícitos es el hecho de que suelen ser usadas para desviar la atención desde la cuestión que se debate a otra que es instalada mediante su uso; el caso más emblemático de este uso lo encontramos en la conocida falacia ad hominem que consiste en que, en lugar de descalificar el argumento del contrario, se descalifica al individuo que lo sostiene, como se describe en la tabla 1.

Tabla 1. Ejemplo de falacia ad hominem

\begin{tabular}{|c|l|}
\hline A: & "Fumar es dañino para la salud porque, como \\
& $\begin{array}{l}\text { lo muestran los estudios médicos de X, Y } \\
\text { y Z, produce una intoxicación de la que el }\end{array}$ \\
B: & $\begin{array}{l}\text { organismo se recupera con dificultad." } \\
\text { "Ud. no puede afirmar eso porque ud. } \\
\text { es un fumador empedernido". }\end{array}$ \\
\hline
\end{tabular}

Fuente: elaboración propia.

Se observa en este ejemplo que, mientras A instala y sostiene la cuestión de lo dañino que es para la salud el hábito de fumar, B no discute este argumento, sino que instala la cuestión de la solvencia moral de A para sostener lo que plantea con lo que la discusión puede derivar fácilmente en la atención a esta nueva cuestión sin que se haya despejado la primera, la que seguirá siendo una cuestión pendiente. Huelga decir que una discusión en la que se recurre a tales recursos no llegará nunca a agotar la cuestión en torno a la cual se organiza originalmente y de ahí la necesidad de evitar su uso como norma práctica general.

En definitiva, la práctica del discurso argumentativo, teniendo en cuenta los elementos señalados, permite reconocer al menos los siguientes elementos básicos: un evento detonador: la existencia de una o más proposiciones susceptibles de controversia; un contexto pragmático de ocurrencia: el debate; una o más cuestiones acerca de las que se debate; unos recursos mediante los cuales se sostiene la cuestión debatida: los argumentos; un o unos preceptos éticos que deben observar quienes discuten y la posibilidad de que, en el curso de la discusión, se recurra a recursos argumentales ilícitos: las falacias.

Estos elementos básicos, considerados en el conjunto de la arquitectura de la que forman parte, se transforman, a su vez, en índices relevantes para la construcción de instrumentos evaluativos que permitan monitorear el desarrollo de las capacidades argumentativas de los alumnos de la enseñanza media, conjurando el sesgo que en esta pudiera introducir la capacidad histriónica o textualizadora de un argumentador dado.

\section{La argumentación en los textos oficiales}

\section{En los estándares de formación}

Los estándares constituyen una extraordinaria herramienta técnica al servicio tanto de la planificación de la formación de profesores como de la evaluación de los resultados de aprendizaje de esa misma formación. En Chile, fueron promulgados en 2012 y contemplan dos campos diferenciados (aunque complementarios) de prescripciones, a saber: el pedagógico y el disciplinario. La forma en 
que son presentados contempla una presentación factual resumida de lo que el profesor debe ser capaz de hacer y una descripción algo más detallada de en qué consiste la capacidad indicada e incorpora (bajo el rótulo de "lo que se manifiesta cuando") un conjunto de índices evidenciales que permiten evaluar la posesión de la capacidad declarada. En las líneas que siguen, nos concentramos en el segundo de estos campos.

Un examen más o menos somero de estos estándares nos lleva a constatar los siguientes elementos relevantes para el objeto que aquí nos ocupa: tanto el estándar 4 (escritura) como los estándares 7, 8 y 9 hacen alguna mención a la argumentación, pero con énfasis distintos y generalmente vinculado a la enseñanza de la escritura, de acuerdo con las convenciones de diversos géneros. La mención más específica y abundante la encontramos, por supuesto, en el estándar 7, que está referido a la comunicación oral y que se muestra según se ilustra ${ }^{7}$ en la figura 1 .

\section{COMUNICACIÓN ORAL}

ESTANDAR 7:

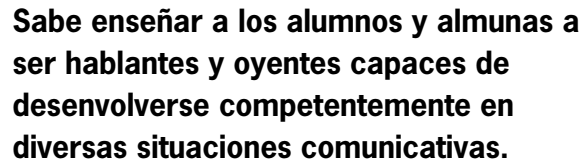

El futuro profesor o profesora sabe enseñar a sus estudiantes a ser hablantes y oyentes capaces de desenvolverse competentemente en diversas situaciones comunicativas del ámbito persnal, laboral académico y ciudadano. Sabe que la comprensión y la producción oral se realizan integradamente y que el lenguaje oral se produce por medio de un código lingüístico con características propias, elementos paralingüísticos y no lingüísticos, que transmiten significados. Comprende que la producción oral se favorece y mejora su calidad cuando se realiza como proceso recursivo que incluye planificar, producir y revisar. Asimismo, comprende que la comprensión oral se favorece y mejora su calidad cuando los oyentes asumen que el emisor tiene una intención y que ellos interpretan el significado, las emociones e ideología del hablante desde sus propios conocimientos y creencias. El futuro profesor o profesora es capaz de modelar interacciones orales de calidad en todo momento del trabajo en aula, reconoce la importancia de familiarizar a los estudiantes con géneros orales formales y conoce estrategias para abordar positivamente eventuales transgresiones de los estudiantes a la lengua estándar o al registro formal. Es capaz de diseñar e implementar actividades motivadoras y desafiantes en que los estudiantes interactúan oralmente con propósitos funcionales promoviendo el desarrollo de habilidades de pensamiento y la construcción colaborativa de conocimientos sobre los temas que se abordan. Sabe generar instancias de reflexión y autoevaluación de los recursos lingüísticos y comunicativos que intervienen en los distintos géneros y registros orales y puede evaluar la producción y comprensión oral de los estudiantes, aplicando criterios pertinentes.

Figura 1. Muestra gráfica de estándares

Fuente: elaboración propia.

7 En donde hemos suprimido los colores del texto original por necesidades de edición. 
Y aunque hay profusas menciones a la interacción, las que podrían augurar un pormenorizado tratamiento de la argumentación toda vez que esta y la conversación coloquial constituyen las manifestaciones más prístinas de la interacción verbal en la cotidianeidad, solo en el número 7 del acápite "Evidencias" se indica lo siguiente:

Conoce y comprende las características de los textos orales formales que se utilizan para argumentar (alocuciones políticas, debate, mesas redondas, juicio oral, etc.). Sabe que ellos:

a) Favorecen las habilidades de pensamiento, en tanto exigen inferir y establecer puntos de vista propios y ajenos, expresar emociones, negociar la divergencia de opiniones, elaborar hipótesis, tesis, razonamientos y conclusiones.

b) Requieren de un vocabulario variado y especializado que permita gestionar porciones extensas de discurso.

c) Presentan recursos lingüísticos, como mostrar la actitud del emisor ("desde mi punto de vista...", "me parece...") o implicar al destinatario ("como todos saben", "miren", "en este punto...") en un esquema lógico que pone en relación premisas, argumentos, contraargumentos y conclusiones.

d) Utiliza estrategias que articulan tipos de razonamiento, como el ejemplo, la comparación, la generalización; y otras que regulan la interacción, como aceptar una parte del razonamiento opositor y refutar los argumentos contrarios. (Mineduc, 2012, pp. 81-82).

Aquí el énfasis está puesto en la dimensión textual de la argumentación, es decir, en uno de sus productos y en los procesos cognitivos que podrían facilitarla, pero hay ausencia tanto de los elementos teóricos básicos como de los procedimientos y competencias implicadas para poder desplegar una conducta argumentativa en la dirección que aquí señalamos como fundamental.

El estándar 4, por su parte y probablemente porque está centrado en la escritura, se enfoca en los propósitos comunicativos de los géneros $y$, aunque menciona la contraargumentación y la discusión, nunca indica procedimiento alguno para implementar estas acciones. Situación similar se plantea en el número 21 de este mismo estándar que se restringe a la planificación de actividades en las que tiene ocasión el debate (sin que se indique nada respecto a qué se entiende por tal y cómo se implementa):

[...] actividades en que los alumnos y alumnas planifican, producen y comprenden textos orales que permiten argumentar, considerando: [...] el uso de estrategias argumentativas adecuadas, tales como formular preguntas retóricas, insertar narraciones breves o anécdotas, atraer la atención, reiterar una idea, dar énfasis, etc. (Mineduc, 2012, p. 83).

Sea porque este estándar se refiere a los conocimientos fundamentales de Literatura o por alguna otra razón, lo cierto es que estos señalamientos giran en torno a la estilística, es decir, del adorno del texto argumentativo, pero claramente no en torno a la argumentación misma.

El estándar 9, y del que podía esperarse un tratamiento más cuidadoso del tema toda vez que la argumentación constituye uno de los objetos predilectos de la investigación lingüística contemporánea en varias de sus subdisciplinas, encontramos una escueta mención estructurada del modo siguiente:

[El profesor] comprende la comunicación oral y escrita como prácticas discursivas sociales que articulan formas verbales y propósitos comunicativos compartidos por las comunidades, lo que da lugar a géneros discursivos constituidos por secuencias, tales como el diálogo, la narración, la exposición y la argumentación. (Mineduc, 2012, p. 87).

Aquí, las ausencias apuntadas arriba se hacen aún más evidentes.

En resumen, pues y habida cuenta de los elementos antes expuestos, resulta claro que los estándares de formación consideran la formación en argumentación de los profesores de lengua castellana y comunicación como algo relevante, pero se observan en ellos unos silencios que no permiten reconocer una organización sistemática de la formación argumentativa de los futuros profesores, lo que -según no 
parece- queda endosado a la responsabilidad de las distintas instituciones formadoras de pedagogos.

\section{En los programas de la enseñanza media}

La enseñanza media chilena y hasta que entre en vigencia su extensión, contemplada en la Ley 20.370, se organiza en cuatro años de formación, para los cuales Mineduc ha promulgado cuatro textos que, bajo el rótulo de "Lenguaje y comunicación. Programa de estudio", ofrece los aprendizajes esperados, contenidos, actividades y formas de evaluación para cada uno de los cuatro años de formación.

Un examen de aquello que los profesores de lengua, formados con las competencias establecidas en los estándares, deben formar en sus alumnos en el específico ámbito que aquí nos interesa, nos ofrece el siguiente panorama que presentamos en orden cronológico de ocurrencia.

En el programa de estudio de primer año medio, ya encontramos una primera mención a la argumentación entre las habilidades que el docente debe promover en sus alumnos en relación con la escritura (en donde se incluye el género ensayo que supone desempeño argumentativo escrito) y en relación con la comunicación oral (en donde se habla de la capacidad de exponer ante otras personas y del desarrollo de la argumentación al formular ideas y opiniones).

La educación media es el lugar en que se refuerzan las habilidades adquiridas en los ciclos básicos, es decir, respetar los turnos de habla y escuchar con atención, adecuarse a la situación comunicativa, utilizar un vocabulario preciso, expresarse con claridad y fundamentar sus opiniones. (Mineduc, 2011, p. 26).

El programa de este nivel se organiza en cuatro unidades (narrativa, poesía, drama, textos no literarios), cuyo desarrollo contempla actividades, contenidos y evaluación para tres macrogrupos de habilidades: lectura, escritura y comunicación oral. De todos estos, el que acoge el tema argumentación es el consagrado a la comunicación oral. En su conjunto, el programa dedica seis aprendizajes esperados (en adelante $\mathrm{AE}$ ) a este contenido y su presencia se muestra más explícita en la formulación de estos AE que en la de las actividades sugeridas para alcanzarlos, aunque estas ofrecen un buen nivel de relación ${ }^{8}$ con los AE que pretenden instanciar. En el aspecto conceptual, es posible reconocer un sustrato fuertemente vinculado al modelo de Toulmin (1958) y lo que parece privilegiar la formación argumentativa en este nivel es que los estudiantes expongan sus ideas siguiendo un orden canónico semejante al postulado por el maestro Korax de Siracusa (Risco, 2015, p. 87) y que, en este caso, corresponde a un esquema que considera tres momentos fundamentales: introducción, cuerpo y cierre, entiendan que se argumenta sobre una cuestión dada, que esta debe sostenerse a lo largo del discurso como requisito de coherencia y, finalmente, que lo afirmado debe estar respaldado por fuentes confiables (respaldos en el modelo de Toulmin). Llama la atención el hecho de que la categoría de argumento se aplique exclusivamente a los respaldos de la tesis y no al conjunto de los elementos incluyendo la misma tesis. Los aspectos éticos más arriba indicados están del todo ausentes.

En el programa de segundo año medio, la situación es similar al caso anterior; es decir, el contenido argumentación se encuentra en el ámbito de la comunicación oral, situación debida -tal vez- al hecho de que la conducta argumentativa es eminentemente un hecho oral; del mismo modo, el contenido se encuentra presente en las cuatro unidades del programa, pero, a diferencia del programa anterior, en este son 11 los AE dedicados al tema y su finalidad parece ser la de reforzar los conceptos y habilidades desarrollados en el programa de primer año. Teóricamente, aparece solo un elemento nuevo: la consideración de la audiencia como factor condicionante de las elecciones del locutor (vocabulario, temas, formas de presentación). La relación de los AE mencionados con el tema argumentación es menos explícita que en el curso anterior y más deducible por medio de un examen de las actividades sugeridas.

En el programa de tercer año medio (que ofrece una versión mejorada del año 2009) ya el tema argumentación ocupa, nominalmente al menos, un

8 Es relevante señalar esto porque (como se verá más adelante) este nivel de relación no es el mismo en otros programas examinados. 
lugar más preponderante toda vez que se le asigna una unidad completa titulada: "Diversidad y conflicto. Argumentación y diálogo" y que el contenido mínimo obligatorio del programa se manifiesta como centrado en la observación del componente argumentativo de los textos, lo que se presenta en los siguientes términos:

Reflexión y comentarios sobre textos periodísticos, cinematográficos, programas radiales y de televisión, avisos y mensajes publicitarios, centrados en la observación del componente argumentativo de ellos y evaluación de los problemas éticos involucrados en la utilización de los procedimientos de persuasión y disuasión (relación de lo verdadero con lo verosímil, de lo bueno con lo deseable, entre otros). (Mineduc, 2009b, p. 13).

Aquí se percibe la presencia de los principales elementos vinculados a la argumentación desde la tradición de la retórica clásica, a saber: argumentación, sus finalidades y consideración ética en torno a la implementación de estas finalidades; aunque la apertura al uso de diversos textos en formatos igualmente diversos augura la aparición de otros conceptos igualmente importantes, aun cuando estos no parezcan previstos en el diseño del programa. Del mismo modo, sobresale en este programa una consideración importante del carácter interactivo del lenguaje, cuestión que se hace relevante cuando su contenido es la argumentación.

En términos generales, la formación anual se organiza en torno al texto argumentativo y no en torno al discurso argumentativo. Esto podría constituir una deficiencia importante ya que este texto, según la dirección desde la que se lo mire, puede introducir tres órdenes de problemas que podríamos resumir a continuación.

Desde la perspectiva que considera al texto argumentativo como un guion previo a su implementación en contexto de interacción oral, estamos ante un objeto que suele parecerse más a un esquema que a un texto propiamente dicho $y$, por tanto, puede conducir a confundir ambos formatos, especialmente si se tiene en cuenta que el gran insumo teórico que suele usarse en estos casos es la planificación de argumentos aislados mediante el esquema ofrecido por el modelo de Toulmin. Esta cuestión, sin embargo, podría verse resuelta mediante un énfasis en la producción de argumentación escrita (texto expositivo), lo que parece ser el énfasis del programa.

Desde la perspectiva que considera al texto argumentativo como un producto del ejercicio de práctica social de la argumentación, a su vez, nos enfrentamos a un objeto que se parece más a una crónica periodística que a un ejercicio interactivo como lo es el de la argumentación que, dicho de paso, se manifiesta de manera privilegiada en el formato oral en el desarrollo de la participación ciudadana que el programa pretende formar.

Por último e independientemente de las objeciones señaladas, queda por resolver el tema de si existe realmente algo que podamos llamar texto argumentativo, toda vez que la descripción de su forma lingüística difícilmente logra aproximarse al reconocimiento de elementos tan pormenorizados como los que permiten describir el texto descriptivo o narrativo y, cuando este esfuerzo es emprendido, suele centrarse más en una descripción del proceso cognitivo-lógico de argumentar (Adam, 1999; Charolles, 1980; Álvarez, 1996; entre otros) que en la presentación de formas lingüísticas específicas que excedan el ámbito de los conectores.

Habida cuenta de estas objeciones, por tanto, hubiera sido esperable que el punto de vista a partir del cual se trata el tema lo constituyera la práctica social de la argumentación, es decir, el discurso argumentativo.

En una visión de conjunto, este curso, aun cuando en comparación con el anterior solo dedica $8 \mathrm{AE}$ al tema argumentación, es posiblemente el que más profundiza (hasta aquí) en ese contenido, ya que incorpora elementos evaluativos tanto de la argumentación escrita como oral generando interesantes oportunidades de profundización para el docente, con un nutrido elenco de actividades sugeridas que, aunque no siempre guardan una relación suficientemente coherente con los AE que pretenden implementar, constituyen una oportunidad para un 
docente con una adecuada formación en argumentación que, justamente en razón de esa adecuada formación, evitará la tentación de concentrarse en los aspectos literarios sobre los que abundan las actividades propuestas. Sin embargo, una amenaza cierta surge del hecho de que un buen desarrollo de este programa implica que los AE de las etapas anteriores se hayan obtenido en un alto nivel.

El programa de cuarto año medio, a su vez, también corresponde a una versión revisada en 2009 y exhibe las siguientes características: se encuentra dividido en cuatro unidades, a saber: tradición y cambio; realidad, deseo y libertad; individuo y sociedad; globalización y diversidad. En estas unidades, la presencia del componente argumentativo se observa ya en la primera de ellas, para la que se indica en los propósitos que:

[...] en cuanto a comunicación oral, se espera que participen activamente en debates, foros, paneles y discusiones usuales de aula, comprendan y evalúen los argumentos usados por otros, y escuchen y analicen un discurso argumentativo pronunciado en una situación pública. En relación con la escritura, que produzcan textos expositivosargumentativos sobre los temas propuestos en los que se manifieste la presencia explícita de una opinión, afirmación o tesis y argumentos, así como el uso de recursos lingüísticos, cognitivos y afectivos, y la utilización de citas y referencias. (Mineduc, 2009c, p. 44).

Los contenidos que se explicitan para conseguir estos propósitos no pasan de indicar aspectos textuales.

En términos generales, este curso presenta ocho $\mathrm{AE}$ referidos a la argumentación, orientados en su mayoría, a la evaluación de la argumentación escrita y oral como también a la producción de discurso argumentativo en contexto público, con pocas novedades teórico-conceptuales, como no sea la incómoda y confusa incorporación de la noción de valor de verdad. Sobresale una notoria inclinación por la implementación de procedimientos persuasivos y una notoria ausencia de medios dirigidos a la convicción.
Aunque estos programas manifiestan un notable enriquecimiento en relación con su versión anterior y que se incluye en ellos la mención de conceptos relevantes para la enseñanza de la argumentación, también es cierto que adolecen de una presentación organizada de esos mismos conceptos que redunde en una comprensión general del fenómeno y las capacidades que se intenta enseñar, máxime cuando muchos de los profesores que deben hacer frente a ese esfuerzo fueron formados en un contexto en el que la argumentación estaba ausente de los programas universitarios de formación de profesores de lengua o se presentaba de manera incipiente.

Un juicio general acerca de estos programas, a partir de los elementos ofrecidos, nos permite afirmar que aquellos manifiestan la importancia de la formación argumentativa de los futuros ciudadanos en la formulación de sus aprendizajes esperados (AE), pero las actividades que sugieren para alcanzar tales aprendizajes son poco pertinentes y contienen suficientes elementos que podrían favorecer un desvío de los esfuerzos hacia la enseñanza de la literatura. Del mismo modo que en el caso de los estándares, no se observa un tratamiento sistemático de los conceptos que pudieran conducir a una adecuada formación de competencias argumentativas que más tarde pudieran enriquecer los debates cotidianos de los futuros ciudadanos, hecho con el que la experiencia supuesta por los alumnos en su sometimiento al programa de formación argumental de la enseñanza media chilena podría no pasar de un recuerdo anecdótico y su desempeño argumentativo en sociedad no pasar de la implementación del procedimiento persuasivo.

\section{Conclusiones}

La relevancia de la formación argumentativa del futuro ciudadano se encuentra suficientemente justificada tanto en la tradición cultural de Occidente como en los textos normativos con los que el Mineduc norma la enseñanza en Chile.

No obstante, una imagen discursiva de lo que los textos ministeriales entienden por argumentación parece más vinculada a la idea de que esta práctica es 
una conducta social predeterminada genéticamente, que el objeto de una formación sistemática, responsabilidad de un sistema escolar que busca preparar para la participación ciudadana efectiva y eficiente.

Los estándares enfatizan la necesidad de la formación argumentativa de los futuros ciudadanos, pero su presentación de los conceptos fundamentales que debe incluir la formación académica de los futuros profesores es todavía exigua e inconexa o, por lo menos, poco sistemática.

La formación argumentativa de los futuros profesores requiere todavía de una concepción más integrada de la conducta argumentativa que, partiendo de una definición consensuada de lo que se debe entender por argumentación, establezca unas competencias básicas a desarrollar en lo futuros ciudadanos y unos índices de evaluación de estas, que resulten compatibles con una noción de argumentación explícita y operativa. Se trata de un desafío que está pendiente.

En la línea de este último señalamiento, es preciso reconocer que todavía se requiere de más esfuerzos por articular los resultados de la investigación académica disciplinar en argumentación con su aplicación o actualización en el ámbito de la formación y desempeño pedagógico.

\section{Referencias}

Adam, J. (1999). Linguistique textuel. Des genres de discours aux textes. París: Natham.

Álvarez, G. (1996). Textos y discursos. Introducción a la lingüística del texto. Concepción, Chile: Editorial de la Universidad de Concepción.

Charolles, M. (1980). Les formes directes et indirectes de l'argumentation. Pratiques, 28, 7-45.

Covarrubias, A. (2003). Introducción a la retórica clásica. Una teoría de la argumentación práctica. Santiago de Chile: Ediciones de la Universidad Católica de Chile.

García, R. (2012). Uso de razón. El arte de razonar, persuadir, refutar. Un programa integral de iniciación a la lógica, el debate y la dialéctica. Kentucky: Lexington.
Jaeger, W. (1946). Paideia. Los ideales de la cultura griega. Vol. I. México D.F.: Fondo de Cultura Económica.

Ministerio de Educación de Chile (Mineduc) (2009a). Ley 20.370 Ley General de Educación. Recuperado de http://www.leychile.cl/Navegar?idNorma=1006043

Ministerio de Educación de Chile (Mineduc) (2009b). Lenguaje y comunicación. Programa de estudio $3^{a}$ medio. Actualización 2009. Santiago de Chile: Unidad de Currículum y Evaluación.

Ministerio de Educación de Chile (Mineduc) (2009c). Lenguaje y comunicación. Programa de estudio cuarto año medio. Santiago de Chile: Unidad de Currículum y Evaluación.

Ministerio de Educación de Chile (Mineduc) (2011). Lenguaje y Comunicación. Programa de Estudio Primer año Medio. Santiago de Chile.

Ministerio de Educación de Chile (Mineduc) (2012). Estandares orientadores para carreras de pedagogía en educación media. Santiago de Chile.

Ministerio de Educación de Chile (Mineduc) (2016a). Presentación. Recuperado de http://media.mineduc. $\mathrm{cl} /$ presentacion-4/

Ministerio de Educación de Chile (Mineduc) (2016b). Orientaciones para la elaboración del plan de formación ciudadana. Santiago de Chile.

Pereda, C. (1994). Vértigos argumentales. Una ética de la disputa. Barcelona: Anthropos.

Perelman, Ch. y Olbrecht-Tyteca, L. (2000). Traité de l'argumentation. 5a. ed. Bruselas: Editions de l'université.

Plantin, Ch. (2002). La argumentación. Barcelona: Ariel.

Risco, E. (2015). Las teorías de la argumentación i: la época fundacional. ALPHA. Revista de Artes, Letras y Filosofía, 40, 81-93.

Rivano, E. (1999). De la argumentación. Santiago de Chile: Bravo y Allende Editores.

Toulmin, S. (1958). The uses of arguments. Cambridge: Cambridge University Press.

Van Eemeren, F., Grootendorst, R., Snoeck, F., Blair, J., Johnson, R., Krabbe, E., Plantin, Ch., Walton, D., Willard, Ch., Woods, J. y Zarefsky, D. (1996). Fundamentals of argumentation Theory. A Handbook of Historical Backgrounds and Contemporary Developements. Nueva Jersey: Lawrence Erlbaum. 
Van Eemeren, F. y Grotendorst, R. (2002). Argumentación, comunicación y falacias. Santiago de Chile: Ediciones de la Universidad Católica de Chile.
Van Eemeren, F. Grootendorst, R. y Snoeck, F. (2006). Argumentación. Análisis, evaluación, presentación. Buenos Aires: Biblos.

\section{Para citar este artículo}

Risco Del Valle, E. (2018). Acerca de la formación argumentativa en el sistema educacional chileno. Folios, 48, 85-99. 